\title{
Identification of an essential binding site for local anesthetics in the 'side pockets' of Kv1 channels
}

\author{
Aytug Kiper ${ }^{1}$, Sarah Stalke ${ }^{1}$, Stefanie Marzian ${ }^{1}$, Mauricio Bedoya ${ }^{2}$, David Ramírez ${ }^{3}$, Alicia \\ De la Cruz ${ }^{4}$, Diego Alberto Peraza ${ }^{5}$, José Márquez Montesinos ${ }^{4}$, Bárbara Arévalo Ramos ${ }^{2}$, \\ Susanne Rinné ${ }^{1}$, Teresa Gonzalez ${ }^{5}$, Carmen Valenzuela ${ }^{5}$, Wendy Gonzalez ${ }^{6}$, and Niels \\ Decher $^{1}$ \\ ${ }^{1}$ University of Marburg, Germany \\ ${ }^{2}$ University of Talca \\ ${ }^{3}$ University of Chile \\ ${ }^{4}$ Instituto de Investigaciones Biomédicas Alberto Sols \\ ${ }^{5}$ Instituto de Investigaciones Biomedicas \\ ${ }^{6}$ Universidad de Talca
}

April 28, 2020

\begin{abstract}
Background and Purpose: Local anesthetics block sodium and a variety of potassium channels. Although previous studies identified a residue in the pore signature sequence together with three residues in the S6 segment as a putative binding site, the precise molecular basis of $\mathrm{Kv}$ potassium channel inhibition by local anesthetics remained unknown. Kv crystal structures predict that some of these residues point away from the central cavity and face into a drug binding site called 'side pockets'. Thus, the question arises whether the binding site of local anesthetics is exclusively located in the central cavity or also involves the 'side pockets'. Experimental Approach: A systematic functional alanine mutagenesis approach, scanning 58 mutants, in concert with in silico docking experiments and molecular dynamics simulations were utilized to elucidate the binding site of bupivacaine and ropivacaine. Key Results: Kv1.5 inhibition by local anesthetics requires binding to the central cavity and the 'side pockets', where the latter requires interactions with residues of the S5 and the backside of the S6 segment. Mutations in the 'side pockets' remove stereoselectivity of Kv1.5 inhibition by bupivacaine. Strikingly, while we found that binding to the 'side pockets' is conserved for the different local anesthetics, the binding mode in the central cavity and the 'side pockets' shows considerable variations. Conclusion and Implications: Local anesthetics bind to the central cavity and the 'side pockets' which provides a crucial key for the molecular understanding of their Kv channel affinity and stereoselectivity, as well as their spectrum of side effects.
\end{abstract}

Identification of an essential binding site for local anesthetics in the 'side pockets' of Kv1 channels

Aytug K. Kiper ${ }^{1, \S}$, Sarah Stalke ${ }^{1, \S}$, Stefanie Marzian ${ }^{1, \S}$, Mauricio Bedoya $^{2,3,8}$, David Ramírez ${ }^{4}$, Alicia de la Cruz ${ }^{5,6}$, Diego A. Peraza ${ }^{5,6}$, José C.E. Márquez Montesinos ${ }^{2}$, Bárbara A. Arévalo Ramos $^{2}$, Susanne Rinné ${ }^{1}$, Teresa Gonzalez ${ }^{5,6,7}$, Carmen Valenzuela ${ }^{5,6, *}$, Wendy Gonzalez ${ }^{2,3, *}$ and Niels Decher ${ }^{1, *}$

${ }^{1}$ Institute for Physiology and Pathophysiology, University of Marburg, Marburg, Germany.

${ }^{2}$ Centro de Bioinformática y Simulación Molecular, Universidad de Talca, Talca, Chile. 
${ }^{3}$ Millennium Nucleus of Ion Channels-Associated Diseases (MiNICAD), Universidad de Talca, Talca, Chile.

${ }^{4}$ Instituto de Ciencias Biomédicas, Universidad Autónoma de Chile, Santiago, Chile.

${ }^{5}$ Instituto de Investigaciones Biomédicas Alberto Sols (CSIC-UAM), Madrid, Spain.

${ }^{6}$ Spanish Network for Biomedical Research in Cardiovascular Research (CIBERCV), Instituto de Salud Carlos III, Madrid, Spain.

${ }^{7}$ Biochemistry Department, School of Medicine, Universidad Autónoma de Madrid, Madrid, Spain.

${ }^{\S}$ Kiper AK, Stalke S, Marzian S and Bedoya M are joint first author.

*Valenzuela C, Gonzalez W and Decher N are corresponding authors.

Running title: Essential binding site for local anesthetics in the Kv 'side pockets'

Prof. Dr. Niels Decher

Institute for Physiology and Pathophysiology

Philipps-University Marburg

Deutschhausstraße 2

35037 Marburg, Germany

$\mathrm{T}:+49-6421-28-62148$

decher@staff.uni-marburg.de

Word count: 3920

\section{ACKNOWLEDGMENTS}

We thank Oxana Nowak for technical assistance. The study was supported, by the Ministerio de Economia y Competitividad (MINECO, Spain) grant SAF2016-75021-R to C.V. and T.G., and Fondo Europeo de Desarrollo Regional (FEDER); the Instituto de Salud Carlos III CIBERCV program (to C.V. and T.G.), the CSIC grant PIE201820E104 (to C.V.) and the CSIC grant 2019AEP148 (to C.V.) and by the Deutsche Forschungsgemeinschaft (DFG) grant DE1482-4/1 to N.D..

\section{AUTHOR CONTRIBUTIONS}

A.K.K., S.S., S.M. and S.R. conducted the electrophysiological experiments in Xenopus oocytes, supervised by N.D.. A.d.l.C. and D.A.P. conducted the electrophysiological experiments in HEK-293 cells, supervised by C.V. and T.G.. M.B., D.R., J.C.E.M.M. and B.A.A.R. generated the in-silico data, supervised by W.G.. S.M. and S.R. generated the mutants. A.K.K., and S.M. analyzed electrophysiological data. A.K.K. performed statistical analyses, generated the figures and contributed to the manuscript writing. N.D., W.G., T.G. and C.V. conceived and designed the experiments. N.D. supervised the whole project and wrote the manuscript.

\section{CONFLICT OF INTEREST}

The authors declare no conflicts of interest.

\section{DATA AVAILABILITY STATEMENT}

The data supporting the findings of this study are available from the corresponding authors upon reasonable request. Some data may not be made available because of privacy or ethical restrictions.

Background and Purpose: Local anesthetics block sodium and a variety of potassium channels. Although previous studies identified a residue in the pore signature sequence together with three residues in the S6 segment as a putative binding site, the precise molecular basis of Kv potassium channel inhibition by local anesthetics remained unknown. Kv crystal structures predict that some of these residues point away from the 
central cavity and face into a drug binding site called 'side pockets'. Thus, the question arises whether the binding site of local anesthetics is exclusively located in the central cavity or also involves the 'side pockets'.

Experimental Approach: A systematic functional alanine mutagenesis approach, scanning 58 mutants, in concert with in silico docking experiments and molecular dynamics simulations were utilized to elucidate the binding site of bupivacaine and ropivacaine.

Key Results: Kv1.5 inhibition by local anesthetics requires binding to the central cavity and the 'side pockets', where the latter requires interactions with residues of the S5 and the backside of the S6 segment. Mutations in the 'side pockets' remove stereoselectivity of Kv1.5 inhibition by bupivacaine. Strikingly, while we found that binding to the 'side pockets' is conserved for the different local anesthetics, the binding mode in the central cavity and the 'side pockets' shows considerable variations.

Conclusion and Implications: Local anesthetics bind to the central cavity and the 'side pockets' which provides a crucial key for the molecular understanding of their Kv channel affinity and stereoselectivity, as well as their spectrum of side effects.

Keywords: local anesthetics, bupivacaine, ropivacaine, Kv1 channels, side pockets, stereoselectivity

\section{Bullet point summary}

\section{What is already known}

- Kv1.5 channels are blocked by local anesthetics in a potent and stereoselective manner.

- 'Side pockets' provide drug binding sites for Kv1 channel blockers.

\section{What this study adds}

- Local anesthetics bind to the central cavity and the 'side pockets' of Kv1.5 channels.

- Binding to 'side pockets' of Kv1.5 determines efficiency and stereoselectivity of local anesthetic inhibition.

\section{What is the clinical significance}

'Side pockets' provide the molecular basis to modulate the efficiency and side effects of antiarrhythmics.

\section{1 | INTRODUCTION}

Local anesthetics block sodium channels by binding to the inactivated state which provides the molecular basis to prevent pain perception. However, local anesthetics also block diverse cardiac ion channels which partially contributes to the cardiotoxicity of local anesthetics (Castle, 1990; Clarkson \& Hondeghem, 1985; Lipka, Jiang et al. , 1998; Valenzuela, Delpon et al. , 1995). Bupivacaine for instance is a long-acting local anesthetic which increases heart rate and arterial blood pressure, reduces cardiac stroke volume and ejection fraction, decreases conductivity and contractility and tends to induce arrhythmias and a long QT syndrome (Clarkson \& Hondeghem, 1985; Kotelko, Shnider et al. , 1984; Sanchez-Chapula, 1988; Scott, Leeet al. , 1989).

Kv1.5 channels that generate the ultrarapid delayed rectifier current $I$ kur regulate atrial action potential durations (Fedida, Wible et al. , 1993; Snyders, Tamkun et al. , 1993) and are major drug targets for the treatment of atrial fibrillation (Decher, Kumar et al., 2006; Kiper, Rinné et al. , 2015). Kv1.5 is blocked by bupivacaine in a potent and stereoselective manner (Franqueza, Longobardo et al. , 1997; Valenzuela, Delpon et al. , 1995). Voltage-gated ion channels share the common feature of a water filled central cavity containing the classical drug binding site in the inner mouth of the channel. The binding sites are mostly formed by two to three amino acid residues of the pore forming helices and one to two residues located in the pore helix. The position of the residues involved in drug binding within these regions are highly conserved from sodium over calcium to different potassium channels (Decher, Kumaret al. , 2006; Decher, Pirard et al. , 2004; Hanner, Greenet al. , 2001; Hockerman, Dilmac et al. , 2000; Mitcheson, Chen et al. , 2000). The recently identified 'side pockets' in voltage-gated potassium $(\mathrm{Kv})$ channels formed by the backsides of the 
S5 and S6 segments together with the S4 and the S4-S5 linker of the neighbouring subunit, serve as a drug binding pocket, providing the molecular basis for an allosteric and irreversible Kv1 specific channel inhibition (Marzian, Stansfeld et al. , 2013).

In early seminal studies probing the pore of Kv channels, it was reported that mutations affecting T441 and T469 of the DrosophilaKv1-related Shaker channel alter open channel block of quaternary ammonium compounds (Choi, Mossman et al. , 1993; Yellen, Jurmanet al. , 1991). Thus, open channel block was proposed to require, as described above for many channels in detail, binding to two sites, one located in the pore loop and one located in the inner mouth of the channel (Baukrowitz \& Yellen, 1996). The two residues identified in these early studies correspond to Kv1.5 residues T479 in the pore signature sequence and T507 of the S6 segment. Furthermore, L510 was discussed as an important drug binding residue in Kv1.5, as studies with the homologue Kv2.1 and Kv3.1 channels showed an altered pharmacology for mutants corresponding to L510 (Aiyar, Nguyen et al. , 1994; Shieh \& Kirsch, 1994). These observations, together with a helical wheel blot analyses led to the misinterpretation that T479, T507, L510 and V514 line the inner cavity of the Kv1.5 channel pore, forming the drug binding site of the channel (Yeola, Rich et al. , 1996). The subsequent studies investigated the role of these putatively pore facing residues as possible binding sites for local anesthetics like bupivacaine (Franqueza, Longobardo et al. , 1997) and benzocaine (Caballero, Moreno et al. , 2002), but also rupatadine (Caballero, Valenzuela et al. , 1999) and irbesartan (Moreno, Caballeroet al. , 2003). The crystal structure of the closely related rKv1.2 channels (Long, Campbell et al. , 2005) revealed however that the amino acids T507, L510 and V514 are not pore facing. In contrast, T507 and L510 perfectly face into the recently identified 'side pockets' that play a crucial role for the development of Kv1 specific blockers (Marzian, Stansfeld et al. , 2013). This led to the question whether bupivacaine and other local anesthetics exclusively interact with the central cavity or also require interactions with the newly identified 'side pockets' (Marzian, Stansfeld et al. , 2013).

To address this question we mapped the binding site of the two local anesthetics bupivacaine and ropivacaine using a systematic functional alanine scanning mutagenesis screen of the S4, S4-S5, S5 and the S6 segments of Kv1.5, combined with in silico docking experiments and molecular dynamics simulations. Our results reveal that local anesthetics do not exclusively bind to the central cavity and that binding to the 'side pockets' is essential for the action of local anesthetics. In addition, we found that a binding of local anesthetics to the central cavity and the 'side pockets' is conserved while the binding modes show considerable variations which might provide the molecular basis to modulate specificity, stereoselectivity and thus the side effects of local anesthetics.

\section{2 | METHODS}

\section{1 | Molecular biology}

PCR-based mutagenesis was used to insert mutations into the hKv1.5 (KCNA5) cDNA. The Kv1.5 cDNA is based on the database entry NM_002234 but differs by two residues (K418R and K565E). Compared to the previous database entry M60451 it includes two additional amino acids in the N-terminus, leading to a shift of the numbering of +2 . The PCR products were fully sequenced (ABI 3100, Applied Biosystems, California, USA). Kv1.5 cDNA was linearized with NheI and complementary cRNA was produced with the mMESSAGE mMACHINE T7-Kit (AM1344 - Ambion, Texas, USA) and its quality was checked by UV spectroscopy (NanoDrop 2000 - Thermo Fisher Scientific, Texas, USA) and gel electrophoresis.

\subsection{Injection and voltage-clamp recordings inXenopus oocytes}

This study has been conducted conforming the guide for the Care and Use of laboratory Animals (NIH Publication 85-23) and the local ethics commission of the "Regierungspräsidium Giessen" approved the experiments using Xenopus frogs (Nasco, Wisconsin, USA) (MR 20/28 Nr. A 23/2017). cRNA for wild-type Kv1.5 or mutant Kv1.5 constructs were injected into isolated stage IV and V Xenopus laevis oocytes as described previously (Streit, Netter et al. , 2011). The oocytes were cultured in bath solution including (in $\mathrm{mM}$ ): $96 \mathrm{NaCl}, 2 \mathrm{KCl}, 1 \mathrm{MgCl}_{2}, 1.8 \mathrm{CaCl}_{2}, 5$ HEPES; $\mathrm{pH} 7.5$ supplemented with $50 \mathrm{mg} / \mathrm{L}$ gentamycine, 274

$\mathrm{mg} / \mathrm{L}$ sodium pyruvate and $88 \mathrm{mg} / \mathrm{L}$ theophylline at $18^{\circ} \mathrm{C}$ for $1-13$ days before the experiments. All mea- 
surements were performed using standard two microelectrode voltage clamp techniques (Stühmer, 1992). Measurements were recorded at room temperature $\left(21-23^{\circ} \mathrm{C}\right.$ ) with a TurboTEC $10 \mathrm{CD}$ amplifier (npi electronic, Tamm, Germany) and a Digidata 1200 Series A/D converter (Molecular Devices, California, USA). Micropipettes with a resistance of $0.5-1.2 \mathrm{M} \Omega$ when filled with $3 \mathrm{mM} \mathrm{KCl}$ were made from borosilicate glass capillaries GB 150TF-8P (Science Products, Hofheim, Germany) and pulled with a DMZ-Universal Puller (Zeitz, Martinsried, Germany). R/S-bupivacaine and ropivacaine were prepared as a $125 \mathrm{mM}$ stock solution in DMSO, stored in a lightproof manner and added to the bath solution just before the recordings. The holding potential was $-80 \mathrm{mV}$. To guarantee a fully regeneration from inactivation a minimum interpulse interval of $10 \mathrm{~s}$ was chosen. The inhibition was calculated at end of a voltage step to $+40 \mathrm{mV}$ for $2 \mathrm{~s}$. Endogenous current component of the oocytes in amount of $150 \mathrm{nA}$ has been subtracted in the analysis to increase the sensitivity of scan. The ratio $I \mathrm{drug} / I \mathrm{ctrl}$ was also determined using this protocol. The current corresponding to the first pulse after a 12 min pulse free period in the presence of drug was divided by the last trace in absence of drug before the pulse free period. The $I V$-protocol which was used to obtain the current-voltage relationship $(I V)$ comprises $10 \mathrm{mV}$ steps in voltage, ranging from $-70 \mathrm{mV}$ to $+70 \mathrm{mV}$ for $200 \mathrm{~ms}$, and a final step to $-40 \mathrm{mV}$ for $300 \mathrm{~ms}$. A Hill plot was used to calculate the half maximal inhibitory concentration $\left(\mathrm{IC}_{50}\right)$. Boltzmann plot was used to calculate the voltage at half maximal activation $\left(\mathrm{V}_{1 / 2}\right)$.

\subsection{Patch clamp recordings with bupivacaine enantiomers and 'side pocket' mutants}

HEK-293 cells were transfected with Kv1.5 cloned in pcDNA3.1 as previously described (Arias, Guizy et al. , 2007; Gonzalez, Navarro-Polanco et al. , 2002). The intracellular pipette filling solution contained $(\mathrm{mM})$ : K-aspartate 80, $\mathrm{KCl} 42$, phosphocreatine $3, \mathrm{KH}_{2} \mathrm{PO}_{4}$ 10, MgATP 3 , HEPES-K 5, and EGTA-K 5, and was adjusted to $\mathrm{pH} 7.25$ with $\mathrm{KOH}$. The bath solution contained (mM): $\mathrm{NaCl} 145, \mathrm{KCl} 4, \mathrm{CaCl}_{2} 1.8$, $\mathrm{MgCl}_{2}$ 1.0, HEPES-Na 10 and glucose 10, and was adjusted to $\mathrm{pH} 7.40$ with $\mathrm{NaOH}$. Both bupivacaine enantiomers were dissolved as stock solutions in deionized MilliQ water at a concentration of $100 \mathrm{mM}$. Kv1.5 currents were recorded at room temperature $\left(21-23^{\circ} \mathrm{C}\right)$ using the whole-cell patch-clamp technique (Hamill, Marty et al. , 1981) with an Axopatch 200B patch-clamp amplifier and a Digidata 1440A A/D converter (Molecular Devices, California, USA). Currents were filtered at $2 \mathrm{kHz}$ (4-pole Bessel filter) and sampled at $4 \mathrm{kHz}$. Micropipettes were pulled from borosilicate glass capillary tubes (Narishige, GD-1, Tokyo, Japan) on a programmable horizontal puller (Sutter Instruments Co., California, USA) and heat-polished with a microforge (Narishige, Japan). Micropipette resistance was 2-4 M 2 . Capacitance and series resistance compensation were optimized, and $80 \%$ compensation of effective access resistance was usually obtained. Cells were held at $-80 \mathrm{mV}$ and $250 \mathrm{~ms}$ pulses between $-80 \mathrm{mV}$ and $+60 \mathrm{mV}$ (in $10 \mathrm{mV}$ steps) were applied at a frequency of $0.1 \mathrm{~Hz}$ in order to avoid accumulation of inactivation. Deactivating tail currents were recorded at $-40 \mathrm{mV}$. The $\mathrm{IC}_{50}$ and Hill coefficients, $n_{H}$, were obtained from fitting the fractional block at various S- or R-bupivacaine concentrations to a Hill plot. The degree of stereoselectivity " $\vartheta$ " was calculated as fold-difference of half maximal inhibitory concentrations between S-bupivacaine and R-bupivacaine.

\section{4 | Homology modelling of the Kv1.5 channel}

The Kv1.5 model previously reported by our group was used to study the binding site for local anesthetics (Marzian, Stansfeld et al. , 2013). Briefly, the crystal structure of the open state Kv1.2-Kv2.1 chimera (PDB code: 2R9R) (Long, Tao et al. , 2007) was used as template. The modelled transmembrane region of Kv1.5 includes the residues 269-526, which correspond to residues 160-417 of the template. Modeller 9v5 (Sali \& Blundell, 1993) was used to create Kv1.5 homology model.

\subsection{Docking of bupivacaine and ropivacaine}

In order to study the binding site and mode of local anesthetics in Kv1.5 ion channel, we used a systematic pipeline that includes docking simulations in each binding site followed by molecular dynamics simulations (MDs) and clustering of conformers. Briefly, two binding sites were selected in the Kv1.5 homology model, the central cavity and one side pocket. We carry out the docking of local anesthetics into only one side pocket because the other three are integrated by the same residues due to the tetrameric nature of the functional channel. The local anesthetics (S-bupivacaine and ropivacaine (INN name implies that it is the S- 
enantiomer)) were sketched and prepared with using LigPrep (Schrödinger Release 2017-1, New York, USA) included in the Maestro suite (Schrödinger Release 2017-1, New York, USA), with the force field OPLS-AA (Kaminski, Friesner et al. , 2001). The nitrogen atom of the piperidine ring was protonated for both ligands. The charges were maintained during the parametrization process before docking and MDs. The docking simulations were performed with Glide (Friesner, Murphyet al. , 2006) using the standard precision scoring function, obtaining 10 poses per docking simulation. The centre of the grid boxes for each binding site was focused using the interacting residues identified by the functional alanine mutagenesis approach for each local anesthetic. All molecular docking runs were performed with the outer box edge of the grid setting as 30 $\AA$, thus ensuring that the binding site residues were included in each grid box. The best poses according to the docking score were analyzed and the one that present the best correlation with the experimental findings were further studied by molecular dynamics simulations.

\section{6 | Molecular dynamics simulations}

The selected poses in the side pocket were triplicated in the other side pockets. The selected pose in the central cavity was also included forming a complex with five ligands, four in each side pocket and one in the central cavity. The protein-ligands complexes were embedded into a pre-equilibrated POPC (1-palmitoyl-2-oleoylsn-glycero-3-phosphocholine) bilayer, then the systems were solvated using the SPC (single point charge) water model. $\mathrm{K}^{+}$ions were placed at sites $\mathrm{S} 2$ and $\mathrm{S} 4$ of the selectivity filter and water molecules at sites S1 and $\mathrm{S} 3 . \mathrm{Cl}^{-}$ions were added to neutralize the system. An ion concentration of $0.15 \mathrm{M} \mathrm{KCl}$ on both sides of the channel was set for the MDs. The systems were equilibrated by $20 \mathrm{~ns}$ in NPT ensemble with positional restraints of $1.0 \mathrm{kcal}^{*} \mathrm{~mol}^{-1 *} \AA^{-2}$ on the secondary structure of the protein, ligands, and ions at the selectivity filter. Temperature and pressure were kept constant at $300 \mathrm{~K}$ and 1.01325 bar, respectively by coupling to a Nose-Hoover Chain thermostat (Cheng \& Merz, 1996) and Martyna-Tobias-Klein barostat (Martyna, Tobias et al. , 1994) with an integration time step of 2 fs. Later, positional restraints were removed, and a molecular dynamics simulation of $100 \mathrm{~ns}$ was done per system using an NPץT (semi-isotropic ensemble) with constant surface tension of 0.0 bar $\AA$ as production. Molecular dynamics simulations were performed used Desmond (Bowers, Chow et al. , 2006) software, v2019-1 and OPLS2005 force field (Shivakumar, Williamset al. , 2010). The same MDs protocol was applied to the Kv1.5 protein (without ligands) as control. From the $100 \mathrm{~ns}-\mathrm{MDs}$ of production, 200 structures from the last $20 \mathrm{~ns}$ (every $0.1 \mathrm{~ns}$ ) were selected to perform the clustering analysis of both ligands (S-bupivacaine and ropivacaine) in each binding site. Protein structure of the selected frames were aligned, and then the cluster analysis was performed by selecting the heavy atoms of the ligand with a defined RMSD cut off $=2 \AA$. Clusters were calculated using the Clustering v2.0.1 plugin (Github June 2014) in the VMD software (University of Illinois, Illinois, USA).

\section{7 | Data and statistical analysis}

The data and statistical analysis comply with the recommendations of the British Journal of Pharmacology on experimental design and analysis in pharmacology (Curtis, Alexander et al. , 2018). No statistical methods were used to predetermine sample sizes and they were estimated based on previous established protocols in the field. No exclusion criteria were pre-established, and no data was excluded from analysis. No randomisation or blinding was performed. Normality of the dataset was tested with a Shapiro-Wilk test and subsequently, equality of variance was tested using either parametric or non-parametric Levene's test. An unpaired Student's t-test was used to probe the significance but for not normally distributed data, the Mann-Whitney U-test. If the variances of the data set were significantly different, statistical significance of the data set was probed with Welch's t-test and for not normally distributed data with Mood's median test. All data are presented as mean \pm s.e.m.. Number of biological replicates (n) are illustrated in the respective figure legends or graphs. Significances are indicated with *, P $<0.01$ in the figures. Data were acquired with pClamp 10 (Molecular Devices, California, USA) and analysed with Clampfit (Molecular Devices, California, USA), Excel 2016 (Microsoft, Washington, USA), OriginPro 2016 (OriginLab, Massachusetts, USA).

\section{8 | Materials}

Gentamicin, sodium pyruvate, theophylline, R/S-bupivacaine, ropivacaine were purchased from Sigma-Aldrich 
(Sigma-Aldrich, Missouri, USA). R-bupivacaine and S-bupivacaine enantiomers were obtained from Astra Zeneca ${ }^{\circledR}$ (London, UK) directly.

\section{3 | RESULTS}

\subsection{Analysis of Kv1.5 inhibition by bupivacaine}

First, we analyzed the inhibition of Kv1.5 channels by racemic R/S-bupivacaine using the Xenopus laevis oocyte expression system (Figure 1a). The $\mathrm{IC}_{50}$ of bupivacaine was $16.9 \pm 3.7 \mu \mathrm{M}$ with a Hill coefficient of $0.76 \pm 0.08$ (Figure 1b). Bupivacaine did not cause a major shift in the voltage-dependence of the Kv1.5 activation curve (Figure 1c). The inhibition of Kv1.5 by bupivacaine was voltage-dependent, as previously described (Gonzalez, Longobardo et al. , 2001; Valenzuela, Delponet al. , 1997). However, in the voltage range of 0 to $+70 \mathrm{mV}$ when the inner gate is primarily in the open state, the block was voltage-independent (Figure 1d). To probe whether the block by bupivacaine requires repetitive channel openings or has a closed state dependence, we measured the currents in the absence of bupivacaine $(I$ ctrl $)$ and after 12 minutes of drug application while the channels were held at $-80 \mathrm{mV}$ in the closed state $\left(I_{\text {bupi }}\right)$ (Figure 1e,f). These recordings revealed that Kv1.5 channels have to be opened before bupivacaine can block the channels and that a rapid open channel block already reaches steady state inhibition within the first test pulse (Figure $1 \mathrm{e}, \mathrm{f})$.

\subsection{Identification of the bupivacaine binding site in the central cavity and side pockets of Kv1.5 channels}

The binding site of local anesthetics in the central cavity of Kv channels was never systematically mapped using an alanine mutagenesis screen. Strikingly, the data from the literature indicates that local anesthetics might also bind to residues outside the central cavity. Therefore, to characterize the bupivacaine binding site in detail, we performed a functional alanine scanning mutagenesis screen (59 mutants) of the pore forming S6 segment and the pore signature sequence, together with the S4 segment, the S4-S5 linker and the S5 segment, as these domains form the 'side pockets' of Kv1.5 channels (Marzian, Stansfeldet al. , 2013). To this end, the inhibition of bupivacaine for the different Kv1.5 alanine mutants was determined by voltageclamp recordings in Xenopus oocytes. Note that endogenous alanine residues were mutated to valine. Under these experimental conditions, $250 \mu \mathrm{M}$ bupivacaine caused an $81.0 \pm 2.0 \%(\mathrm{n}=8)$ inhibition of wild-type Kv1.5. The working hypothesis of this experimental approach was that a mutant channel lacking its regular amino acid side chain should have a reduced potency for bupivacaine. The potency of bupivacaine was significantly reduced for T480A of the pore signature sequence and for I502A, L510A, V512A and V516A of the S6 segment (Figure 1g). T480, V512 and V516 are pore facing amino acids belonging to the classical drug binding site for high affinity blockers in the central cavity of Kv1.5 channels (Decher, Kumar et al. , 2006; Decher, Pirard et al. , 2004; Marzian, Stansfeld et al. , 2013; Strutz-Seebohm, Gutcher et al. , 2007), whereas I502 faces into fenestrations connecting the central cavity with the side pockets and L510 directly faces into the 'side pockets' (Marzian, Stansfeld et al. , 2013). Moreover, we found that three residues (T479, T507 and V514) previously proposed to contribute to the local anesthetic binding site (Caballero, Moreno et al. , 2002; Franqueza, Longobardoet al. , 1997), did not significantly alter bupivacaine binding. In contrast, T480 of the pore signature sequence plays a much more pronounced role for local anesthetic binding than the initially proposed T479 (Caballero, Moreno et al. , 2002; Franqueza, Longobardoet al. , 1997). Thus, the alanine scan of the S6 segment already revealed revisited and also unexpected results for the binding site of the local anesthetic bupivacaine (Figure S1).

Most importantly, these results support the idea that bupivacaine interacts with the classical binding site in the central cavity and the selectivity filter as well as with parts of the 'side pockets' of the Kv1.5 channel. While we reported that mutations in the S4 and S4-S5 linker do not affect inhibition by bupivacaine (Figure 1g, dashed bars and box described by (Marzian, Stansfeld et al. , 2013)), the alanine scan of the S5 domain revealed significantly reduced bupivacaine potency for four mutant channels (Figure 1g). The four residues that we identified in S5, L436, F439, F440 and I443, directly face into the 'side pockets', similar as L510 of the S6 segment. These data further support that bupivacaine binds to the central cavity and the 'side 
pockets' of Kv1.5 channels.

As it was previously proposed that local anesthetics preferentially bind to the inactivated state of sodium channels, we tested for a correlation between inhibition and the intrinsic inactivation properties of the mutants. However, plotting the extent of C-type inactivation of single mutants against the respective inhibition, did not reveal such a correlation (Figure 1h). Thus, the residues identified by our alanine scan most likely exhibit a reduced affinity, due to an impaired drug binding.

\subsection{Binding to both, the central cavity and the 'side pockets' is an essential prerequisite for stereoselective inhibition of Kv1.5 by bupivacaine}

Bupivacaine causes a strong stereoselective inhibition of Kv1.5 (Arias, Guizy et al., 2007; Franqueza, Longobardo et al. , 1997; Valenzuela, Delpon et al. , 1995) with the R- enantiomer being 9-fold ( $\vartheta$ ) more potent (Figure 2a,f). Mutants at T507, L510 and V514 were previously reported to modulate the stereoselectivity of bupivacaine inhibition (Franqueza, Longobardo et al. , 1997). Due to the Kv1.2 crystal structure we know by now that these residues are located at the backside of S6 and face into the 'side pockets'. Strikingly, we found that the S5 mutants F440A and I443A drastically reduced the stereoselectivity of bupivacaine (Figure 2b-f) with an almost complete loss of stereoselectivity for I443A (Figure 2d,f).

Mutants at T479 located in the pore signature sequence facing the central cavity did not affect the stereoselectivity of bupivacaine inhibition (Franqueza, Longobardo et al. , 1997). In addition, we found that T479A does not cause a major reduction in bupivacaine inhibition (Figure 1g), presumably as the threonine side chain is not perfectly facing into the central cavity. In contrast, mutating the neighbouring residue T480 caused a drastic reduction in bupivacaine affinity (Figure 1g) and an almost complete loss of stereoselectivity inhibition of Kv1.5 by bupivacaine (Figure 2e,f). These experiments show that residues in the central cavity and the 'side pockets' are crucial determinants for the stereoselectivity inhibition of Kv1.5 by bupivacaine. The fact that residues in the 'side pockets' determine bupivacaine affinity and stereoselectivity strongly argues for a binding of this local anesthetic to the 'side pockets' of Kv1.5 channels.

3.4 Identification of the ropivacaine binding site in the central cavity and 'side pockets' of Kv1.5 channels

Next we probed the binding site of ropivacaine (which is the S-enantiomer) that also blocks Kv1.5 channels (Valenzuela, Delponet al. , 1997), albeit with a reduced stereoselectivity of inhibition $(\vartheta=2.5$-fold) (Longobardo, Delpon et al. , 1998). The following experiments were performed to probe whether the differential binding sites for these two local anesthetics actually determine the reduced stereoselectivity and affinity of ropivacaine compared to bupivacaine. First, ropivacaine had an $\mathrm{IC}_{50}$ of $141.6 \pm 6.7 \mu \mathrm{M}$ with a Hill coefficient of $1.1 \pm 0.02$ (Figure 3a,b) and thus was, as previously reported, less potent than bupivacaine. Ropivacaine caused a voltage-independent inhibition in the depolarized voltage range of 0 to $+70 \mathrm{mV}$ (Figure 3d) and blocked Kv1.5 channels with an open state affinity (Figure 3e,f), similar as bupivacaine.

The potency of ropivacaine was reduced for the S6 segment mutants T480A, L510A, V512A and V516A that either face the pore or the 'side pockets', similar as for bupivacaine (Figure $3 \mathrm{~g}$ versus Figure 1g). For the I502A mutant that faces into fenestrations that connect the central cavity with the 'side pockets', the effects were somewhat more pronounced and with L506A and I508A we identified two additional pore facing residues to be relevant for the inhibition by ropivacaine. Thus, also for ropivacaine the results support the idea that the drug interacts with the classical binding site in the central cavity and the selectivity filter, as well as with parts of the 'side pockets' of Kv1.5.

Strikingly, we also identified for ropivacaine L436, F439, F440 and I443 in the S5 segment, residues that face into the 'side pockets' (Figure 3g). However, with L437 we identified one additional residue in the S5 segment that faces into fenestrations connecting the 'side pockets' to the central cavity, similar as I502 does (Marzian, Stansfeld et al. , 2013). Most importantly, ropivacaine requires interactions with residues of the S4 segment (L413) and the S4-S5 linker (L420), residues that were previously reported to interact with Psora-4 bound to the 'side pockets' (Marzian, Stansfeld et al. , 2013). Thus, ropivacaine binds to the central cavity 
and the 'side pockets' of Kv1.5 channels. In addition, ropivacaine, which has a reduced stereoselectivity and affinity, must utilize a different binding site or mode in the 'side pockets'.

Also for ropivacaine, plotting the extent of C-type inactivation of single mutants against their respective inhibition, did not reveal a correlation between the inactivation properties of the mutants and their ropivacaine affinity (Figure 3h). Thus, the residues identified by our alanine scan most likely exhibit a reduced affinity, due to an impaired drug binding.

\subsection{Model of the bupivacaine and ropivacaine binding mode in the central cavity and the 'side pockets' of Kv1.5}

To determine the binding mode of bupivacaine and ropivacaine in Kv1.5 channels, we used in silico docking experiments and molecular dynamics (MD) simulations of charged S-bupivacaine and ropivacaine, respectively, using a Kv1.5 homology model based on the rKv1.2-Kv2.1 chimera crystal structure (Figure 4). After docking of Kv1.5 in complex with five ligands, one in the central cavity and one in each 'side pocket', we performed $100 \mathrm{~ns}$ molecular dynamics (MD) simulations and selected 200 complexes for each system (a pose every $0.1 \mathrm{~ns}$ from the last $20 \mathrm{~ns}$ ) to perform a clustering analysis. According to this analysis we identified highly populated clusters in the central cavity and the 'side pockets', for both local anesthetics (Figure 4 and Supplementary Table 1). This indicates that in general, both local anesthetics adopt a fairly stable conformation during the simulation in the central cavity and in the 'side pockets', since the population of the clusters provides a measure for the stability of the 'drug to receptor' interaction. The most populated clusters contained in all cases more than $50 \%$ of the total number of 200 conformers. For both local anesthetics, some clusters were even formed by all or almost all ligand conformers (central cavity and in some of the 'side pockets', see Supplementary Table 1).

The proposed binding mode of bupivacaine in the central cavity is illustrated in Figure 4b, illustrating a cluster containing 198 out of 200 conformers. The ropivacaine binding mode is illustrated in Figure 4c, containing 199 out of the 200 conformers. Both local anesthetics interact with Kv1.5 in a similar mode (Figure 4b,c). For both compounds the most populated cluster (Figure 4b,c) provided the basis for interactions with the residues identified in the alanine mutagenesis scan of the S6 segment (Figure 1g, 3g), except for L510 as this residue is facing into the 'side pockets'.

Figure 4d,f illustrates the two most likely binding modes of bupivacaine in the 'side pockets' of Kv1.5, representing the two most occupied clusters (containing 200 and 199 conformers). The two most occupied 'side pockets' clusters (containing 196 and 182 conformers) derived from the MD simulations with ropivacaine are illustrated underneath (Figure 4e,g). Note that the two most occupied clusters of bupivacaine are located at the opposite end of the 'side pockets' (Figure 4d,f) compared to ropivacaine (Figure 4e,g). Consistent with our alanine-scanning mutagenesis, ropivacaine interacts with the S4 segment, residues of the proximal S4-S5 linker and the backside of the S5 and S6 segment of a neighbouring subunit (Figure 4e,g). In contrast, bupivacaine does not bind next to the S4 segment and is interacting with residues of the S5 segment of the same subunit (Figure 4d,f), supporting our initial alanine mutagenesis screen of the S4 and S4-S5 linker in which we did not observe any effects on bupivacaine inhibition (Marzian, Stansfeldet al. , 2013) (Figure 1g). Thus, both local anesthetics bind to the central cavity and the 'side pockets', albeit binding to the 'side pockets' can occur in two different regions (Figure 5) which might be involved in tuning stereoselectivity and affinity of Kv1 channel inhibition by local anesthetics.

\section{4 | DISCUSSION}

We found that binding to the central cavity and 'side pockets' is conserved among local anesthetics, while in the 'side pockets' binding can occur in different modes. Nevertheless, the local anesthetics bound to the newly identified sites in the 'side pockets' are essentially determining the potency of the drugs, as mutations in the 'side pockets' strongly diminish the affinity of both compounds, bupivacaine and ropivacaine. Besides tuning the apparent affinity, the 'side pockets' are contributing to the enantioselectivity present for the inhibition of Kv1.5 by bupivacaine. The regular Hill factor of the dose-response curve of around one for the Kv1.5 inhibition indicates that local anesthetics must independently bind to the central cavity and the 'side pockets', 
with no cooperativity between the two sites. Yet, for an efficient drug block or in the case of bupivacaine for a stereoselective inhibition, binding to both sites, the central cavity and the 'side pockets', appears mandatory. How many of the four 'side pockets' need to be occupied by local anesthetic for an efficient channel inhibition remains however an open question. This question is hard to address experimentally, especially as we found that designing concatameric channels often results in altered biophysical and pharmacological properties.

Kv1.5 open channel blockers expose varying voltage- and use-dependencies or cooperativity of inhibition. Tikhonov \& Zhorov hypothesized that this might be caused by a common mechanism, meaning that the compounds either form a blocking particle itself by a charged moiety of the drug or by binding of neutral drugs to a potassium at the $\mathrm{S}_{5}$ site in the cavity, using different stoichiometries for the formation of the respective blocking particle complex (Tikhonov \& Zhorov, 2014). From this common position underneath the selectivity filter $\left(\mathrm{S}_{5}\right.$ site) hydrophobic parts of the drugs were proposed to either remain in the central cavity or to laterally protrude into the side fenestrations to interact with I502, a residue relevant for many Kv1.5 blockers. However, we now found that not only Psora-4, but also ropivacaine and bupivacaine bind to the 'side pockets' to reach I502 from the other side of the fenestrations. Considering the current study, several drugs were now reported to utilize the 'side pockets' to alter drug affinity or in the case of bupivacaine also stereoselectivity. Therefore, the discussed variabilities in the kinetics or cooperativity of Kv1 channel inhibition might not be exclusively caused by the formation of different charged drug potassium complexes in the central cavity, but also or even exclusively by an additional drug binding in the 'side pockets'.

An open question in the field is how local anesthetics cause a stereoselective inhibition of Kv1.5 channels. Kvss1.3 which binds to the central cavity of Kv1.5 (Decher, Gonzalez et al. ,2008) reduces stereoselectivity of bupivacaine inhibition (Arias, Guizy et al. , 2007). Strikingly, the $\vartheta$ value is only reduced from about nine to four (Arias, Guizy et al. , 2007), despite that Kvß1.3 interacts with all the directly pore facing residues that we have also identified as binding sites for local anesthetics, including T480, I508, V512 and V516 (Decher, Kumar et al. , 2005). These data indicate that there are other residues outside the central cavity that co-determine the stereoselectivity of Kv1.5 inhibition by local anesthetics. Consistently, our mutagenesis data with T480A, F440A and I443A indicate that stereoselective inhibition of Kv1.5 by bupivacaine is determined by residues in the pore and the 'side pockets', also further supporting that efficient inhibition by local anesthetics actually requires binding to both distinct binding sites. For local anesthetics it has been shown that reducing the length of the alkyl side chain at the piperidine ring reduces affinity and stereoselectivity (Longobardo, Delpon et al. , 1998). Strikingly, ropivacaine, which has a propyl instead of a butyl side chain compared to bupivacaine and is exhibiting a reduced affinity and almost no stereoselectivity for Kv1.5 inhibition (Longobardo, Delpon et al. , 1998), actually maps to a different binding site in the 'side pockets' than bupivacaine. The ropivacaine binding site determined by in silico docking experiments and MD simulations involves interactions with the S4 segment and the proximal S4-S5 linker and a binding to the S5 segment of a neighboring channel subunit. At the ropivacaine binding site, the interactions with the S5 segment residues F440 and I443 that are involved in determining the stereoselective inhibition of bupivacaine, can easily be accomplished and thus these residues are presumably accessible for both enantiomers. In contrast, bupivacaine maps to the other site of the 'side pockets' from where it appears that an interaction with the S5 residues F440 and I443 from the same subunit might be more restricted and only possible or preferred for one of the enantiomers. The differential set of residues that we have mapped for the two local anesthetics in the 'side pockets' provides the basis for future studies to carefully elaborate how binding to the 'side pockets' contributes to stereoselective channel inhibition of local anesthetics. Unfortunately, the above mentioned hypothesis currently remains unaddressed as in silico docking experiments and MD simulations are methodologically not powerful enough to resolve how this relative small stereoselectivity is achieved for a blocker that also displays a rather low potency of channel inhibition.

In earlier studies, it was thought that T479 of the pore signature sequence, together with T507, L510 and V514 of the S6 segment face the inner pore of Kv1.5 (Yeola, Rich et al. , 1996), as mutations at these sites altered the pharmacology of the channel. The crystal structures of the bacterial rKv1.2 channel (Long, Campbell et al. , 2005) revealed that T507, L510 and V514 are not pore facing and instead face into 'side pockets' that we have recently described as drug binding site for the Kv1 channel blocker Psora-4 (Marzian, 
Stansfeldet al. , 2013). Consistently, we have identified in an alanine-scanning approach a novel binding site for local anesthetics which is located outside of the central cavity. Unfortunately, most previous studies used only these limited set of mutants to analyze the putative drug binding sites of quinidine (Yeola, Rich et al. , 1996), benzocaine (Caballero, Moreno et al. , 2002), bupivacaine (Caballero, Moreno et al. , 2002; Franqueza, Longobardo et al. , 1997), rupatadine (Caballero, Valenzuela et al. , 1999) or irbesartan (Moreno, Caballero et al. , 2003). Therefore, it is possible that besides Psora-4 and local anesthetics, many more drugs, including the ones mentioned above, are actually utilizing the 'side pocket' to cause or modulate Kv1 channel inhibition.

Our results reveal that local anesthetics do not exclusively bind to the central cavity and that binding to the 'side pockets' is essential for the action of local anesthetics, providing the molecular basis to modulate specificity, stereoselectivity and thus the spectrum of side effects of local anesthetics.

\section{References}

Aiyar J, Nguyen AN, Chandy KG, \& Grissmer S (1994). The P-region and S6 of Kv3.1 contribute to the formation of the ion conduction pathway.Biophys J, 67(6), 2261-2264.

Arias C, Guizy M, David M, Marzian S, Gonzalez T, Decher N, \& Valenzuela C (2007). Kvbeta1.3 reduces the degree of stereoselective bupivacaine block of Kv1.5 channels. Anesthesiology, 107(4), 641-651.

Baukrowitz T, \& Yellen G (1996). Two functionally distinct subsites for the binding of internal blockers to the pore of voltage-activated $\mathrm{K}^{+}$channels. Proc Natl Acad Sci USA, 93(23), 13357-13361.

Bowers KJ, Chow DE, Xu H, Dror RO, Eastwood M, Gregersen BA, ... Sacerdoti FD (2006). Scalable Algorithms for Molecular Dynamics Simulations on Commodity Clusters. ACM/IEEE SC 2006 Conference (SC'06), IEEE, 2006(43.

Caballero R, Moreno I, Gonzalez T, Valenzuela C, Tamargo J, \& Delpon E (2002). Putative binding sites for benzocaine on a human cardiac cloned channel (Kv1.5). Cardiovasc Res, 56(1), 104-117.

Caballero R, Valenzuela C, Longobardo M, Tamargo J, \& Delpon E (1999). Effects of rupatadine, a new dual antagonist of histamine and platelet-activating factor receptors, on human cardiac Kv1.5 channels. $\mathrm{Br}$ J Pharmacol, 128(5), 1071-1081.

Castle NA (1990). Bupivacaine inhibits the transient outward $\mathrm{K}^{+}$current but not the inward rectifier in rat ventricular myocytes. J Pharmacol Exp Ther, 255(3), 1038-1046.

Cheng A, \& Merz KM (1996). Application of the Nosé-Hoover Chain Algorithm to the Study of Protein Dynamics. J Phys Chem,100(1927-1937.

Choi KL, Mossman C, Aube J, \& Yellen G (1993). The internal quaternary ammonium receptor site of Shaker potassium channels. Neuron, 10(3), 533-541.

Clarkson CW, \& Hondeghem LM (1985). Mechanism for bupivacaine depression of cardiac conduction: fast block of sodium channels during the action potential with slow recovery from block during diastole.Anesthesiology, 62(4), 396-405.

Curtis MJ, Alexander S, Cirino G, Docherty JR, George CH, Giembycz MA, ... Ahluwalia A (2018). Experimental design and analysis and their reporting II: updated and simplified guidance for authors and peer reviewers. Br J Pharmacol, 175(7), 987-993.

Decher N, Gonzalez T, Streit AK, Sachse FB, Renigunta V, Soom M, ... Sanguinetti MC (2008). Structural determinants of Kvbeta1.3-induced channel inactivation: a hairpin modulated by $\mathrm{PIP}_{2} . E M B O \mathrm{~J}, 27(23)$, 3164-3174.

Decher N, Kumar P, Gonzalez T, Pirard B, \& Sanguinetti MC (2006). Binding site of a novel Kv1.5 blocker: a "foot in the door" against atrial fibrillation. Mol Pharmacol, 70(4), 1204-1211. 
Decher N, Kumar P, Gonzalez T, Renigunta V, \& Sanguinetti MC (2005). Structural basis for competition between drug binding and Kvbeta 1.3 accessory subunit-induced N-type inactivation of Kv1.5 channels. Mol Pharmacol, 68(4), 995-1005.

Decher N, Pirard B, Bundis F, Peukert S, Baringhaus KH, Busch AE, ... Sanguinetti MC (2004). Molecular basis for Kv1.5 channel block: conservation of drug binding sites among voltage-gated $\mathrm{K}^{+}$channels. $J$ Biol Chem, 279(1), 394-400.

Fedida D, Wible B, Wang Z, Fermini B, Faust F, Nattel S, \& Brown AM (1993). Identity of a novel delayed rectifier current from human heart with a cloned $\mathrm{K}^{+}$channel current. Circ Res,73(1), 210-216.

Franqueza L, Longobardo M, Vicente J, Delpon E, Tamkun MM, Tamargo J, ... Valenzuela C (1997). Molecular determinants of stereoselective bupivacaine block of hKv1.5 channels. Circ Res, 81(6), 1053-1064.

Friesner RA, Murphy RB, Repasky MP, Frye LL, Greenwood JR, Halgren TA, ... Mainz DT (2006). Extra precision glide: docking and scoring incorporating a model of hydrophobic enclosure for protein-ligand complexes. J Med Chem, 49(21), 6177-6196.

Gonzalez T, Longobardo M, Caballero R, Delpon E, Tamargo J, \& Valenzuela C (2001). Effects of bupivacaine and a novel local anesthetic, IQB-9302, on human cardiac K+ channels. J Pharmacol Exp Ther, 296(2), 573583.

Gonzalez T, Navarro-Polanco R, Arias C, Caballero R, Moreno I, Delpon E, .. Valenzuela C (2002). Assembly with the Kvbeta1.3 subunit modulates drug block of hKv1.5 channels. Mol Pharmacol, 62(6), 1456-1463.

Hamill OP, Marty A, Neher E, Sakmann B, \& Sigworth FJ (1981). Improved patch-clamp techniques for high-resolution current recording from cells and cell-free membrane patches. Pflugers Arch, 391(2), 85-100.

Hanner M, Green B, Gao YD, Schmalhofer WA, Matyskiela M, Durand DJ, ... Garcia ML (2001). Binding of correolide to the Kv1.3 potassium channel: characterization of the binding domain by site-directed mutagenesis.Biochemistry, 40(39), 11687-11697.

Hockerman GH, Dilmac N, Scheuer T, \& Catterall WA (2000). Molecular determinants of diltiazem block in domains IIIS6 and IVS6 of L-type $\mathrm{Ca}^{2+}$ channels. Mol Pharmacol, 58(6), 1264-1270.

Kaminski GA, Friesner RA, Tirado-Rives J, \& Jorgensen WL (2001). Evaluation and reparametrization of the OPLS-AA force field for proteins via comparison with accurate quantum chemical calculations on peptides.J Phys Chem B, 105(6474-6487.

Kiper AK, Rinné S, Rolfes C, Ramirez D, Seebohm G, Netter MF, ... Decher N (2015). Kv1.5 blockers preferentially inhibit TASK-1 channels: TASK-1 as a target against atrial fibrillation and obstructive sleep apnea? Pflugers Arch, 467(5), 1081-1090.

Kotelko DM, Shnider SM, Dailey PA, Brizgys RV, Levinson G, Shapiro WA, .. Rosen MA (1984). Bupivacaineinduced cardiac arrhythmias in sheep.Anesthesiology, 60(1), 10-18.

Lipka LJ, Jiang M, \& Tseng GN (1998). Differential effects of bupivacaine on cardiac K channels: role of channel inactivation and subunit composition in drug-channel interaction. J Cardiovasc Electrophysiol, 9(7), 727-742.

Long SB, Campbell EB, \& Mackinnon R (2005). Crystal structure of a mammalian voltage-dependent Shaker family K ${ }^{+}$channel. Science, 309(5736), 897-903.

Long SB, Tao X, Campbell EB, \& MacKinnon R (2007). Atomic structure of a voltage-dependent $\mathrm{K}^{+}$channel in a lipid membrane-like environment. Nature, 450(7168), 376-382.

Longobardo M, Delpon E, Caballero R, Tamargo J, \& Valenzuela C (1998). Structural determinants of potency and stereoselective block of hKv1.5 channels induced by local anesthetics. Mol Pharmacol, 54(1), 
$162-169$.

Martyna GJ, Tobias DJ, \& Klein ML (1994). Constant pressure molecular dynamics algorithms. J Chem Phys, 101(4177-4189.

Marzian S, Stansfeld PJ, Rapedius M, Rinne S, Nematian-Ardestani E, Abbruzzese JL, ... Decher N (2013). Side pockets provide the basis for a new mechanism of $\mathrm{Kv}$ channel-specific inhibition. Nat Chem Biol,9(8), 507-513.

Mitcheson JS, Chen J, Lin M, Culberson C, \& Sanguinetti MC (2000). A structural basis for drug-induced long QT syndrome. Proc Natl Acad Sci U S A, 97(22), 12329-12333.

Moreno I, Caballero R, Gonzalez T, Arias C, Valenzuela C, Iriepa I, . . Delpon E (2003). Effects of irbesartan on cloned potassium channels involved in human cardiac repolarization. J Pharmacol Exp Ther,304(2), 862873.

Sali A, \& Blundell TL (1993). Comparative protein modelling by satisfaction of spatial restraints. $J$ Mol Biol, 234(3), 779-815.

Sanchez-Chapula J (1988). Effects of bupivacaine on membrane currents of guinea-pig ventricular myocytes. Eur J Pharmacol, 156(3), 303-308.

Scott DB, Lee A, Fagan D, Bowler GM, Bloomfield P, \& Lundh R (1989). Acute toxicity of ropivacaine compared with that of bupivacaine.Anesth Analg, 69(5), 563-569.

Shieh CC, \& Kirsch GE (1994). Mutational analysis of ion conduction and drug binding sites in the inner mouth of voltage-gated $\mathrm{K}^{+}$channels. Biophys J, 67(6), 2316-2325.

Shivakumar D, Williams J, Wu Y, Damm W, Shelley J, \& Sherman W (2010). Prediction of Absolute Solvation Free Energies using Molecular Dynamics Free Energy Perturbation and the OPLS Force Field. $J$ Chem Theory Comput, 6(5), 1509-1519.

Snyders DJ, Tamkun MM, \& Bennett PB (1993). A rapidly activating and slowly inactivating potassium channel cloned from human heart. Functional analysis after stable mammalian cell culture expression. $J$ Gen Physiol, 101(4), 513-543.

Streit AK, Netter MF, Kempf F, Walecki M, Rinné S, Bollepalli MK, ... Decher N (2011). A specific twopore domain potassium channel blocker defines the structure of the TASK-1 open pore. J Biol Chem,286(16), 13977-13984.

Strutz-Seebohm N, Gutcher I, Decher N, Steinmeyer K, Lang F, \& Seebohm G (2007). Comparison of potent Kv1.5 potassium channel inhibitors reveals the molecular basis for blocking kinetics and binding mode. Cell Physiol Biochem, 20(6), 791-800.

Stühmer W (1992). Electrophysiological recording from Xenopus oocytes. Methods Enzymol, 207(319-339.

Tikhonov DB, \& Zhorov BS (2014). Homology modeling of Kv1.5 channel block by cationic and electroneutral ligands. Biochim Biophys Acta, 1838(3), 978-987.

Valenzuela C, Delpon E, Franqueza L, Gay P, Snyders DJ, \& Tamargo J (1997). Effects of ropivacaine on a potassium channel (hKv1.5) cloned from human ventricle. Anesthesiology, 86(3), 718-728.

Valenzuela C, Delpon E, Tamkun MM, Tamargo J, \& Snyders DJ (1995). Stereoselective block of a human cardiac potassium channel (Kv1.5) by bupivacaine enantiomers. Biophys J, 69(2), 418-427.

Yellen G, Jurman ME, Abramson T, \& MacKinnon R (1991). Mutations affecting internal TEA blockade identify the probable pore-forming region of a $\mathrm{K}^{+}$channel. Science, 251(4996), 939-942.

Yeola SW, Rich TC, Uebele VN, Tamkun MM, \& Snyders DJ (1996). Molecular analysis of a binding site for quinidine in a human cardiac delayed rectifier $\mathrm{K}^{+}$channel. Role of S6 in antiarrhythmic drug binding. 
Circ Res, 78(6), 1105-1114.

\section{Figure legends}

FIGURE 1 . Characterization of the inhibition of Kv1.5 channels by bupivacaine and mapping of the binding site. (a) Representative voltage-clamp recordings of Kv1.5 expressed in Xenopus laevisoocytes before and after application of $250 \mu \mathrm{M} \mathrm{R} / \mathrm{S}$-bupivacaine. (b) Dose-response curve for Kv1.5 inhibition by R/S-bupivacaine. (c) G/V-relationship of Kv1.5 in the absence and presence of $250 \mu \mathrm{M} \mathrm{R} / \mathrm{S}$-bupivacaine, respectively. (d) Analyses of the voltage-dependence of Kv1.5 inhibition by $250 \mu \mathrm{M} \mathrm{R} / \mathrm{S}$-bupivacaine in the voltage range of -20 to $+70 \mathrm{mV}$. (e) Analyses of the use-dependence of Kv1.5 inhibition by R/S-bupivacaine $(250 \mu \mathrm{M})$. The inset illustrates representative Kv1.5 currents measured before and the first two pulses directly after a $12 \mathrm{~min}$ pulse free period, during which the drug was washed-in while the cells were held at $-80 \mathrm{mV}$. (f) Ratio of $I$ drug to $I$ control determined for the first voltage-step, derived from (e). (g) Alanine-scanning of the S4 segment, the S4-S5 linker, as well as the S5 and S6 segments. Analyses of the inhibition by 250 $\mu \mathrm{M} R / \mathrm{S}$-bupivacaine for the mutants and the respective wild-type channels. The values illustrated for the inhibition of S4 segment and S4-S5 linker mutants (dashed bars and box) was previously reported by Marzian et al. , 2013. (h) Lack of correlation between the inactivation properties of the mutants tested and their apparent affinity. The inactivation properties of the mutants were analyzed as percentage of inactivation over a time course of $1 \mathrm{~s}$ at $+40 \mathrm{mV}$ and were derived from the study by Marzian et al. , 2013. Significances are indicated with $*, \mathrm{P}<0.01$.

FIGURE 2. Stereoselectivity of Kv1.5 inhibition by bupivacaine is determined by residues in the central cavity and the 'side pockets'. (a) Whole-cell patch clamp recordings of wild-type Kv1.5 recorded in HEK-293 cells, before and after the application of $50 \mu \mathrm{M}$ R-bupivacaine or S-bupivacaine, respectively. (b) Patch clamp recordings of I443A, before and after the application of $250 \mu \mathrm{M}$ R-bupivacaine or S-bupivacaine, respectively. (c-e) Dose-response curves of Kv1.5 inhibition by R-bupivacaine and S-bupivacaine for the (c) F440A, (d) I443A and (e) T480A mutants. Dashed lines indicate the respective dose-response curves for wild-type Kv1.5 inhibition by R-bupivacaine and S-bupivacaine described in the literature by Arias et al. , 2007. (f) Degree of the stereoselectivity ( $\vartheta$ value) for wild-type Kv1.5 and the F440A, I443A and T480A mutants.

FIGURE 3 . Characterization of the inhibition of Kv1.5 channels by ropivacaine and mapping of the binding site. (a) Representative voltage-clamp recordings of Kv1.5 expressed in Xenopus laevisoocytes before and after application of $1 \mathrm{mM}$ ropivacaine. (b) Dose-response curve for Kv1.5 inhibition by ropivacaine. (c) G/V-relationship of Kv1.5 in the absence and presence of $1 \mathrm{mM}$ ropivacaine, respectively. (d) Analyses of the voltage-dependence of Kv1.5 inhibition by $1 \mathrm{mM}$ ropivacaine in the voltage range of -20 to $+70 \mathrm{mV}$. (e) Analyses of the use-dependence of Kv1.5 inhibition by $1 \mathrm{mM}$ ropivacaine. The inset illustrates representative Kv1.5 currents measured before and the first two pulses directly after a 12 min pulse free period, during which the drug was washed-in while the cells were held at $-80 \mathrm{mV}$. (f) Ratio of $I$ drug to $I$ control determined for the first voltage-step, derived from (e). (g) Alanine-scanning of the S4 segment, the S4-S5 linker, as well as the S5 and S6 segments. Analyses of the inhibition by $1 \mathrm{mM}$ ropivacaine for the mutants and the respective wild-type channels. (h) Lack of correlation between the inactivation properties of the mutants tested and their apparent affinity. The inactivation properties of the mutants were analyzed as percentage of inactivation over a time course of $1 \mathrm{~s}$ at $+40 \mathrm{mV}$ and were derived from the study by Marzianet al. , 2013. Significances are indicated with ${ }^{*}, \mathrm{P}<0.01$.

FIGURE 4. Binding modes of S-bupivacaine and ropivacaine in a Kv1.5 homology model. (a) Top and side view of a Kv1.5 homology model based on the Kv1.2-Kv2.1 chimera crystal structure (PDB code: 2R9R). (b,c) Illustration of the binding mode of (b) S-bupivacaine and (c) ropivacaine in the central cavity of Kv1.5. Depicted are the most populated clusters determined by 100 ns molecular dynamics simulations. (d,e) Illustration of the most populated cluster or binding mode in the 'side pockets' for (d) S-bupivacaine and for (e) ropivacaine. (f,g) Illustration of the second most populated cluster or binding mode in the 'side pockets' for (f) S-bupivacaine and for (g) ropivacaine.

FIGURE 5. Differential binding mode of bupivacaine and ropivacaine in the 'side pockets' of Kv1.5. (a) 
Top view of a Kv1.5 homology model based on the Kv1.2-Kv2.1 chimera crystal structure (PDB code: 2R9R) with S-bupivacaine and ropivacaine bound to the four different 'side pockets'. (b) Side view illustrating the differential binding modes of bupivacaine and ropivacaine. Neighbouring subunits can be distinguished as they are illustrated in gray versus dark gray.

\section{Figure 1}

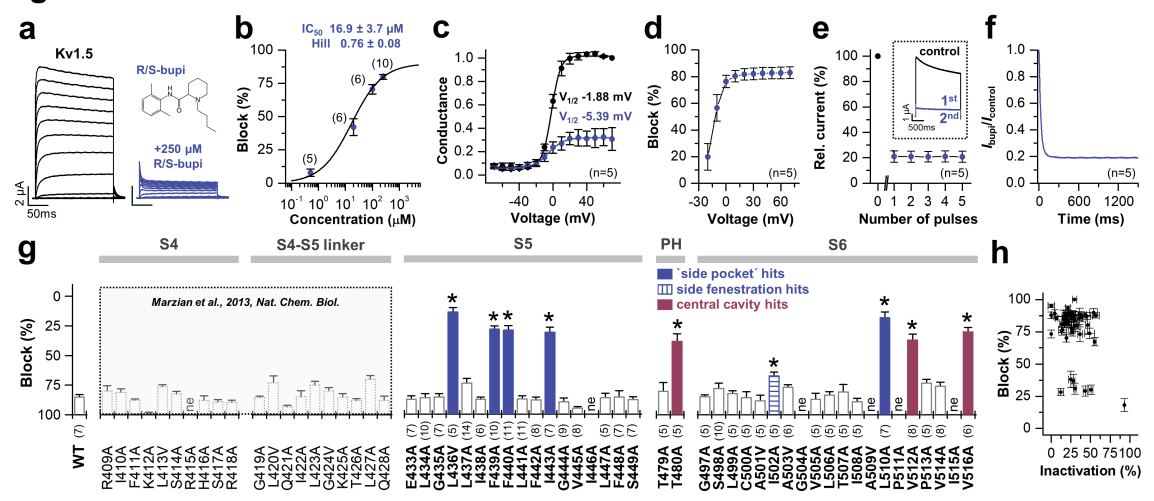

\section{Figure 2}

a
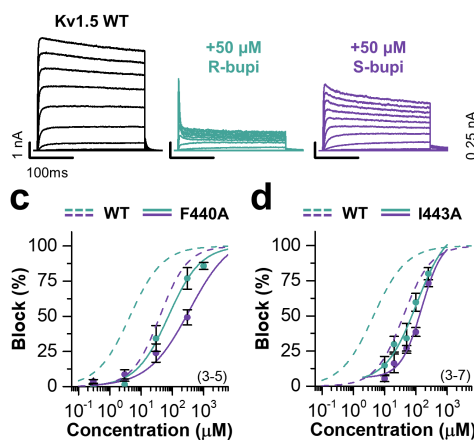

b

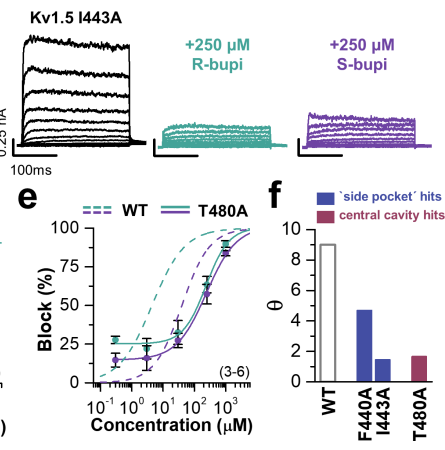

Figure 3

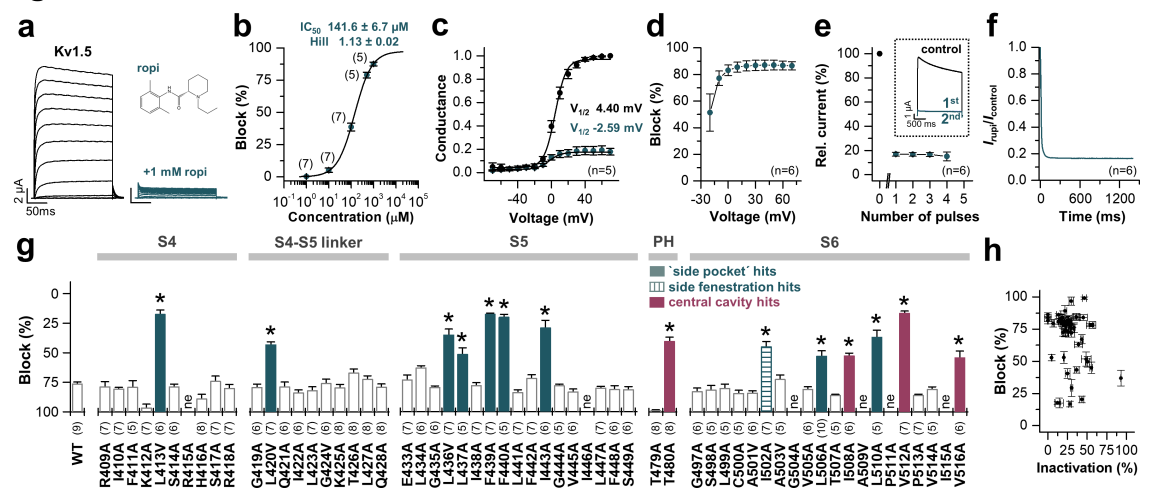


Figure 4
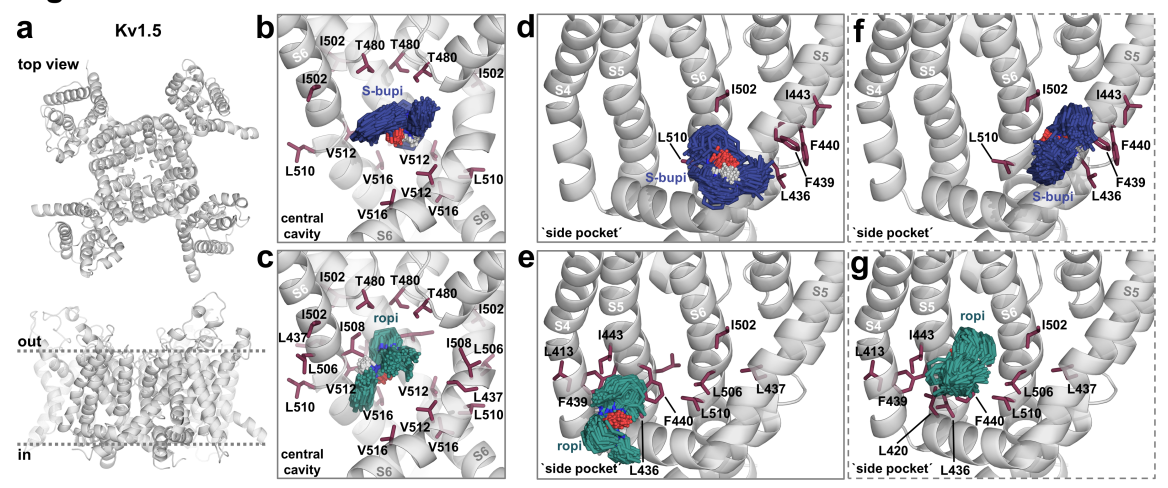

Figure 5

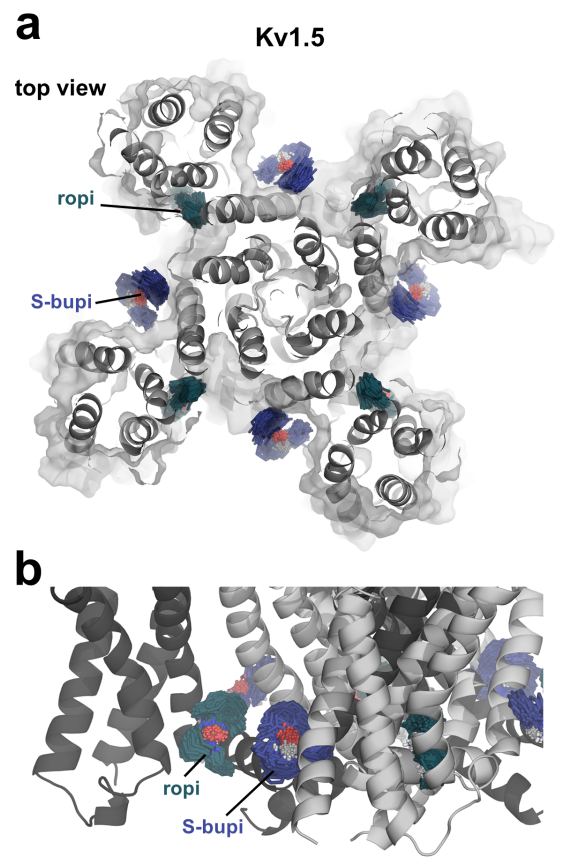

